\title{
Acute Type A Dissection during Pregnancy with Marfan's Syndrome
}

\author{
Christian Heim ${ }^{1}$ Philipp P. Müller ${ }^{1} \quad$ Michael Weyand $^{1} \quad$ Frank Harig ${ }^{1}$ \\ ${ }^{1}$ Department of Cardiac Surgery, Friedrich-Alexander-University \\ Erlangen-Nuremberg, Erlangen, Germany \\ Thorac Cardiovasc Surg Rep 2021;10:e18-e21. \\ Address for correspondence Christian Heim, MD, MHBA, Department \\ of Cardiac Surgery, Friedrich-Alexander-University Erlangen- \\ Nuremberg, Krankenhausstr. 12, 91054 Erlangen, Germany \\ (e-mail: Christian.heim@fau.de).
}

\author{
Abstract \\ Keywords \\ - aortic dissection \\ - Marfan \\ - pregnancy
}

Background Marfan's syndrome (MFS) is a connective tissue disorder, caused by a mutation in the $F B N-1$ gene and affecting multiple organ systems including the vascular system. During pregnancy, women with MFS have an increased risk of aortic dissection due to changes in the cardiovascular system.

Case Description We present a 39-year-old woman that suffered from an acute aortic dissection in week 33 of her third pregnancy who urgently required lifesaving surgery. Three-dimensional reconstruction of computed tomography scan was performed with unborn child in utero.

Conclusion Monitoring of patients with suspected or confirmed MFS appears essential and interdisciplinary treatment and prevention strategies are required.

\section{Introduction}

Marfan's syndrome (MFS) is an autosomal dominant disorder caused by mutations in the $F B N-1$ gene, encoding for fibrillin 1 , a glycoprotein which is a major component of the extracellular matrix. ${ }^{1}$ Manifestations of this disease can be variable, but the musculoskeletal, the ocular, and the cardiovascular systems are most commonly affected in patients with MFS. ${ }^{2}$ There is also evidence that the mutation type in the $F B N-1$ gene is associated with the degree of severity of cardiovascular manifestations. ${ }^{3}$ Aortic complications such as dissections occur in more than one-third of MFS patients. $^{4}$

In the state of pregnancy, women experience changes in the cardiovascular system. Hypervolemia due to an increased blood volume leads to cardiac hypertrophy which is considered a physiological change in pregnant women. ${ }^{5}$ Additionally, an increase in steroid hormone levels may lead to a dilation of the aorta, due to a decline of elastic fibers and mucopolysaccharides in the tunica media of the aorta ${ }^{6}$ and pregnant women with MFS are especially affected by these changes. Despite the previously described correlation between pregnancy and acute aortic dissection in patients with $\mathrm{MFS}^{7,8}$ the implementation of guidelines for the treatment of affected women ${ }^{9}$ in terms of prevention, surveillance, and treatment strategies are often inadequately. Therefore, we want to present a dramatic case of a 39-year-old woman, who suffered from acute type A aortic dissection in week 33 of her third pregnancy.

\section{Case Presentation}

A 39-year-old woman with previous diagnosis of MFS was admitted to an external hospital with acute chest pain and shortness of breath in week 33 of her third pregnancy. After computed tomography was performed to confirm the suspected diagnosis of acute type A dissection, the patient was transferred to our university cardiac surgery department. At the same time, the obstetricians unit of our hospital was informed for emergency cesarean section. When the patient received

November 5, 2020 accepted after revision November 24, 2020
DOI https://doi.org/ $10.1055 / \mathrm{s}-0040-1722705$. ISSN 2194-7635.

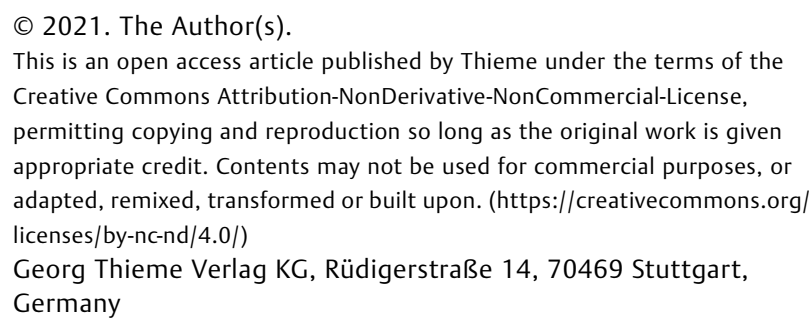




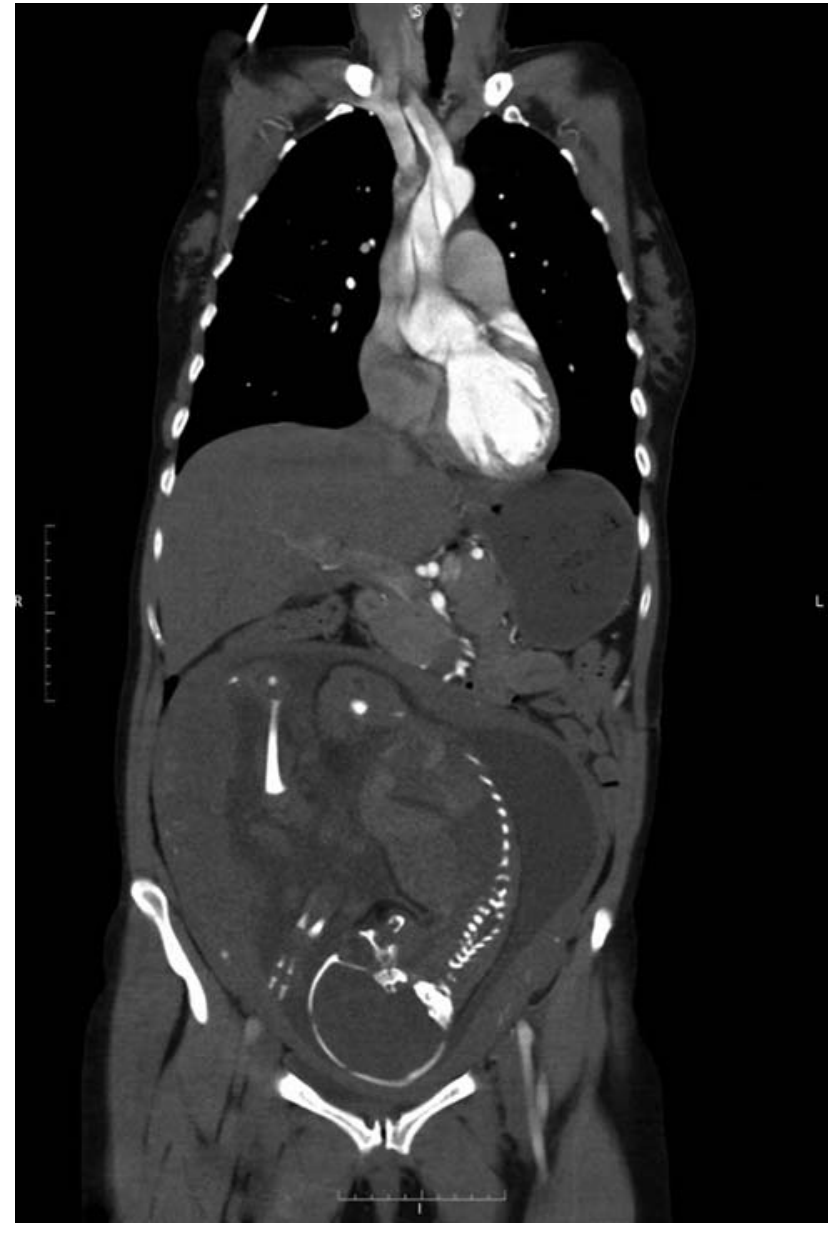

Fig. 1 Computed tomography scan confirming suspected aortic dissection with unborn child in utero. The spiraled dissection reached from the aortic valve annulus to the iliac bifurcation.

was prepared for surgery in the operating room, ventricular fibrillation occurred and cardiopulmonary resuscitation (CPR) was performed. After computed tomography was performed to confirm the suspected diagnosis of acute type A dissection (-Figs. 1 and $\mathbf{2}$ ).

Under continued CPR, median sternotomy was performed to provide access to the heart. Cannulation of the ascending aorta and the right atrium was used for the establishment of cardiopulmonary bypass (CPB) in systemic hypothermia. Subsequently, the proximal part of the dissected brachiocephalic artery was cannulated to provide brain perfusion. When cardioplegia was applied directly through the coronary ostia, an additional rupture of the right coronary artery was noticed. The ascending aorta, the aortic valve, and the left coronary button were excised and a mechanical valve conduit was implanted. Following this, aortocoronary venous bypass was performed to the right coronary artery. Selective antegrade cerebral perfusion was performed to examine the extend of the dissection regarding the aortic arch. After clamping of the brachiocephalic trunk, neuromonitoring showed that cerebral oxygen saturation remained on a stable level. After removing of the aortic cannula, the entire aortic arch showed to be dissected, including the brachiocephalic trunk, the left common

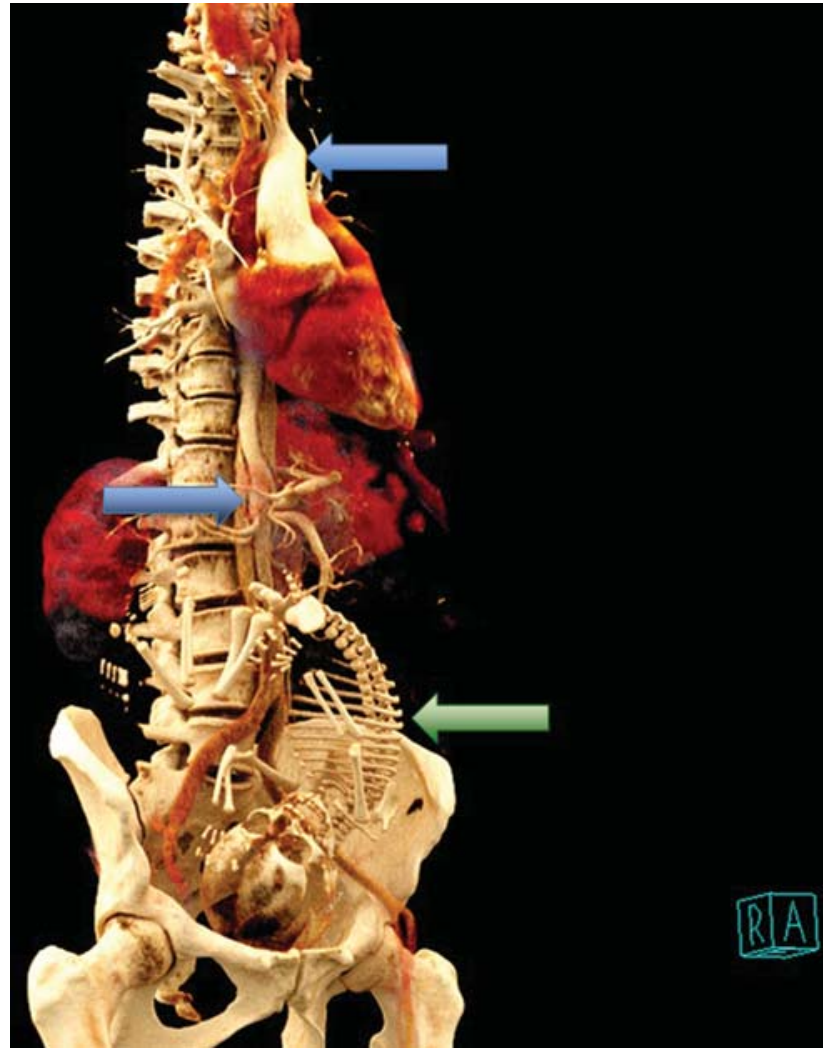

Fig. 2 Three-dimensional reconstruction showing aortic dissection reaching from ascending aorta (light blue arrow) to the infrarenal aorta (dark blue arrow). The unborn child was in utero (green arrow) at this moment and was delivered via cesarian section during cardiopulmonary resuscitation of the mother.

carotid artery, and the left subclavian artery. Brachiocephalic vessels were controlled by tourniquets. After that, the affected vessels as well as the aortic arch were resected. Subsequently, a graft-to-descending aorta anastomosis with a hybrid prosthesis was performed. After clamping of the prosthesis, the neoaorta was cannulated for CPB. Following this, a graft-to-graft anastomosis, reconstruction of the brachiocephalic trunk, the left common carotid artery, and the left subclavian artery and the corresponding anastomoses to the hybrid prosthesis were performed. Despite intensive inotropic support, termination of CPB was not possible; therefore, a central venoarterial extracorporeal membrane oxygenation (ECMO) was implanted. Under these conditions, the patient was transferred to the intensive care unit (ICU). Despite this extensive surgical procedure and an elaborated postoperative ICU stay, it was possible to discharge the patient after ECMO weaning into neurological rehabilitation. The clinical course of the newborn child was uneventful.

\section{Discussion}

Untreated aortic aneurysm in women with MFS, who are especially vulnerable in pregnancy can be fatal as aortic dissection is associated with high rates in morbidity and mortality. ${ }^{10}$ Additionally, the fetal mortality rate is up to $50 \%$ in case of an aortic dissection. ${ }^{11}$ Nevertheless, it was possible to save both 
Table 1 International recommendations summarized from the following studies

\begin{tabular}{|c|c|}
\hline \multicolumn{2}{|c|}{ Counseling and management of patients with MFS during pregnancy ${ }^{9,16,18}$} \\
\hline $\begin{array}{l}\text { Risk assessment prior to } \\
\text { or during pregnancy }\end{array}$ & $\begin{array}{l}\text { Advise patients with MFS not to become pregnant, if }{ }^{9} \\
\text { - Ascending aorta }>45 \mathrm{~mm} \text { or } \\
\text { - Ascending aorta }>40 \mathrm{~mm} \text { and family history of dissection } \\
\text { Counseling about the risk of aortic dissection prior to and during pregnancy }{ }^{9,16} \\
\text { - No dilatation, intermediate risk of maternal mortality (maternal cardiac event rate, } 10-19 \%)^{18} \\
\text { - } 40-45 \mathrm{~mm} \text {, significantly increased risk of maternal mortality; requires expert counseling } \\
\text { (maternal cardiac event rate, } 19-27 \%)^{18} \\
\text { - }>45 \mathrm{~mm} \text {, extremely high risk of maternal mortality; termination should be sensitively } \\
\text { discussed if pregnancy occurs (maternal cardiac event rate, } 40-100 \%)^{18}\end{array}$ \\
\hline Diagnostic management & $\begin{array}{l}\text { - Echocardiographic measurements every } 4-12 \text { wk of the ascending aortic dimensions are } \\
\text { recommended to detect aortic expansion until birth and } 6 \text { months postpartum }{ }^{9,16} \\
\text { - For imaging of dilated distal ascending aorta, aortic arch, or descending aorta, MRI } \\
\text { (without gadolinium) is recommended }{ }^{16}\end{array}$ \\
\hline Therapeutic management & $\begin{array}{l}\text { Medical } \\
\text { - Strict blood pressure control }{ }^{16}, \beta \text {-blocker therapy throughout pregnancy } \\
\text { Cardiac } \\
\text { - Consider prophylactic surgery when aortic dilatation and/or advancing aortic valve } \\
\text { regurgitation are documented } \\
\text { Delivery where cardiothoracic surgery is available }{ }^{16} \\
\text { - Ascending aorta }<40 \mathrm{~mm} \text {, vaginal delivery is feasible } \\
\text { - Ascending aorta }>45 \mathrm{~mm} \text { or (history of) dissection, cesarean delivery should be considered } \\
\text { Type A aortic dissection during pregnancy } \\
\text { - Multidisciplinary care including obstetricians, fetal care, cardiothoracic/vascular } \\
\text { surgeon, and anesthetists. Optimal perioperative management including full maternal } \\
\text { and fetal monitoring, attention to cardiopulmonary bypass, pulsatile perfusion, etc. } \\
\text { - Viable fetus: cesarian section followed by aortic surgery } \\
\text { - Nonviable fetus: aortic surgery with fetus in uterus }\end{array}$ \\
\hline
\end{tabular}

Abbreviation: MFS, Marfan's syndrome.

mother and child, despite the increased perioperative risk. ${ }^{12}$ This case shows dramatically that a high alertness regarding cardiovascular surveillance in the state of pregnancy is crucial for these patients. Despite the fact that the women in our case was pregnant for the third time and MFS as well as aortic dilation was diagnosed previously, there was a lack in education about the risks accompanied by pregnancy regarding aortic dissection. Periodically monitoring changes in the aortic diameter could have been performed during pregnancy. This shows that a multidisciplinary approach, involving gynecologist, cardiologist, and cardiac surgeons is mandatory to monitor affected patients. Medical therapy can be essential to decrease the risk for aortic dissection in pregnant women. While some drugs are contraindicated in pregnancy (angiotensin-converting enzyme inhibitors), some $\beta$ and $\alpha$ adrenergic blockers can prescribed safely and are associated with a decline in dissection rates. ${ }^{13}$ Additionally, if a critical aortic diameter is reached during pregnancy, planned termination or elective aortic surgery can be discussed. ${ }^{14}$ Hereby, the risk for acute aortic dissection could be decreased, which in turn would decrease mortality rates for the mother and the child at the same time.

In terms of women with MFS contemplating pregnancy, prepregnancy counseling is advised to evaluate the specific risk for each patient. ${ }^{15}$ Genetic analyses of the mutation type may be helpful to assess the risk for cardiovascular complications as studies have shown that certain mutation types are associated with an increased risk in cardiovascular events. ${ }^{3}$ Magnetic resonance imaging to detect preexisting aneurysms can be a useful tool to further create an individual risk profile for women with MFS. If aortic dilation is diagnosed, prophylactic aortic replacement should be discussed. Current guidelines recommend that aortic replacement should be considered in patients with an aortic diameter of $5.5 \mathrm{~cm}$ even if they have no symptoms ${ }^{16}$ and studies show that aortic replacement can be performed safely, especially in young patients with no preexisting comorbidities. $^{17}$

\section{Conclusion}

Acute type A dissection in pregnant women with MFS is associated with high rates regarding morbidity and mortality. Despite a detailed understanding of changes in the cardiovascular system in the state of pregnancy and the well-known association of aortic events in patients with MFS, there are yet no universal recommendations for affected women. - Table 1 summarizes international recommendations regarding counseling and management of patients with MFS prior to and during pregnancy. Close monitoring for pregnant women with MFS and a detailed education prior to pregnancy are crucial measures. Prophylactic aortic replacement may be discussed and can be performed safely in young patients without comorbidities. 


\section{Authors' Contribution}

Concept/design and critical revision of article were done by C.H. and M.W. Also, C.H. contributed in funding. Data interpretation and drafting article were done by F.H. and P. P.M., respectively.

\section{Institutional Review Board Waiver and Patient Consent} Statement

Patient signed informed consent related to clinical course, therefore and due to its retrospective nature of the educational case report, the Institutional Review Board was waived.

Funding

This publication was supported by Deutsche Forschungsgemeinschaft and University of Erlangen Foundation within the funding programme Open Access Publishing.

\section{Conflict of Interest}

None declared.

\section{References}

1 Sakai LY, Keene DR, Renard M, De Backer J. FBN1: the diseasecausing gene for Marfan syndrome and other genetic disorders. Gene 2016;591(01):279-291

2 Bitterman AD, Sponseller PD. Marfan syndrome: a clinical update. J Am Acad Orthop Surg 2017;25(09):603-609

3 Franken R, Teixido-Tura G, Brion M, et al. Relationship between fibrillin-1 genotype and severity of cardiovascular involvement in Marfan syndrome. Heart 2017;103(22):1795-1799

4 Groth KA, Stochholm K, Hove H, et al. Aortic events in a nationwide Marfan syndrome cohort. Clin Res Cardiol 2017;106(02): 105-112

5 Chung E, Leinwand LA. Pregnancy as a cardiac stress model. Cardiovasc Res 2014;101(04):561-570

6 Smith K, Gros B. Pregnancy-related acute aortic dissection in Marfan syndrome: a review of the literature. Congenit Heart Dis 2017; 12(03):251-260

7 Lind J, Wallenburg HC. The Marfan syndrome and pregnancy: a retrospective study in a Dutch population. Eur J Obstet Gynecol Reprod Biol 2001;98(01):28-35
8 Katsuragi S, Ueda K, Yamanaka K, et al. Pregnancy-associated aortic dilatation or dissection in Japanese women with Marfan syndrome. Circ J 2011;75(11):2545-2551

9 Regitz-Zagrosek V, Roos-Hesselink JW, Bauersachs J, et al;ESC Scientific Document Group. 2018 ESC guidelines for the management of cardiovascular diseases during pregnancy. Eur Heart J 2018;39(34):3165-3241

10 Adams JN, Trent RJ. Aortic complications of Marfan's syndrome. Lancet 1998;352(9142):1722-1723

11 Goland S, Elkayam U. Cardiovascular problems in pregnant women with Marfan syndrome. Circulation 2009;119(04):619-623

12 Arnoni RT, Arnoni AS, Bonini RC, et al. Risk factors associated with cardiac surgery during pregnancy. Ann Thorac Surg 2003;76(05): $1605-1608$

13 Shores J, Berger KR, Murphy EA, Pyeritz RE. Progression of aortic dilatation and the benefit of long-term beta-adrenergic blockade in Marfan's syndrome. N Engl J Med 1994;330(19):1335-1341

14 Lansman SL, Goldberg JB, Kai M, Tang GH, Malekan R, Spielvogel D. Aortic surgery in pregnancy. J Thorac Cardiovasc Surg 2017;153 (02):S44-S48

15 Cox DA, Ginde S, Kuhlmann RS, Earing MG. Management of the pregnant woman with Marfan syndrome complicated by ascending aorta dilation. Arch Gynecol Obstet 2014;290(04):797-802

16 Hiratzka LF, Bakris GL, Beckman JA, et al;American College of Cardiology Foundation/American Heart Association Task Force on Practice Guidelines American Association for Thoracic Surgery American College of Radiology American Stroke Association Society of Cardiovascular Anesthesiologists Society for Cardiovascular Angiography and Interventions Society of Interventional Radiology Society of Thoracic Surgeons Society for Vascular Medicine. 2010 ACCF/AHA/AATS/ACR/ASA/SCA/SCAI/SIR/STS/ SVM guidelines for the diagnosis and management of patients with Thoracic Aortic Disease: a report of the American College of Cardiology Foundation/American Heart Association Task Force on Practice Guidelines, American Association for Thoracic Surgery, American College of Radiology, American Stroke Association, Society of Cardiovascular Anesthesiologists, Society for Cardiovascular Angiography and Interventions, Society of Interventional Radiology, Society of Thoracic Surgeons, and Society for Vascular Medicine. Circulation 2010;121(13):e266-e369

17 Kunihara T, Aicher D, Asano M, et al. Risk factors for prophylactic proximal aortic replacement in the current era. Clin Res Cardiol 2014;103(06):431-440

18 Fletcher. et al. Inherited thoracic aortic disease: new insights and translational targets. Circulation 2020;141(19):1570-1587 